\title{
Prevalence of Tuberculosis and Rifampicin Resistance Among Patients Seeking Medical Care in Nasarawa State, North Central Nigeria
}

\author{
Egbe Kingsley ${ }^{1}$, Ike Anthony C. ${ }^{1,}$, , Aleruchi Chuku ${ }^{2}$ \\ ${ }^{1}$ Department of Microbiology, University of Nigeria, Nsukka, Enugu State, Nigeria \\ ${ }^{2}$ Department of Microbiology, Federal University Lafia, Nasarawa State, Nigeria
}

Email address:

anthonyc.ike@unn.edu.ng (Ike A. C.)

*Corresponding author

\section{To cite this article:}

Egbe Kingsley, Ike Anthony C., Aleruchi Chuku. Prevalence of Tuberculosis and Rifampicin Resistance Among Patients Seeking Medical Care in Nasarawa State, North Central Nigeria. Science Journal of Public Health. Vol. 4, No. 3, 2016, pp. 214-218.

doi: $10.11648 /$ j.sjph.20160403.18

Received: April 18, 2016; Accepted: April 25, 2016; Published: May 13, 2016

\begin{abstract}
Tuberculosis (TB), caused by Mycobacterium tuberculosis remains a clinical and public health challenge in Nigeria. This study was carried out to determine the prevalence of $M$. tuberculosis and rifampicin resistance in patients from five selected health facilities in Nasarawa State, Nigeria. The HIV serostatus and the CD4+ count of seropositive individuals were also determined. Using a combination of Zhiel Neelsen staining and WHO approved GeneXpert MTB/Rif device for $M$. tuberculosis and rifampicin resistance, the presence of $M$. tuberculosis in sputum samples was detected and confirmed. A total of 346 samples were analysed, out of which M. tuberculosis was detected in $91(26.3 \%)$ samples and rifampicin resistance in 6 $(6.6 \%)$ of the positive samples. HIV prevalence was found to be $46.8 \%$, HIV and TB co-infection $39.6 \%$, while $83.3 \%$ of the rifampicin resistance was from HIV positive cases. Tuberculosis was found to be decreasing with increase in CD4 count. Statistically, there was a significant relationship $(\mathrm{P}<0.05)$, between level of education, HIV, CD4 count and TB occurrence. However, no significant relationship $(\mathrm{P}>0.05)$ between gender, occupation and age with TB was observed. Our results show that tuberculosis still remains endemic in Nasarawa state with a high prevalence of HIV and that the confluence of HIV and tuberculosis increased the rate of rifampicin resistance. Provision of Tuberculosis diagnostics with the capacity to detect TB drug resistance beyond rifampicin is highly recommended.
\end{abstract}

Keywords: Tuberculosis, Rifampicin Resistance, GeneXpert MTB/Rif, Human Immunodeficiency Virus, CD4 Count

\section{Introduction}

Tuberculosis is an infectious bacterial disease caused by Mycobacterium tuberculosis, which most commonly affects the lungs [1]. It is transmitted from person to person via droplets from the throats and lungs of people with the active respiratory disease and occurs in every part of the world [2]. Tuberculosis has been causing great suffering to human beings throughout recorded history. Even two decades after introduction of directly observed treatment, short-course (DOTS) strategy, tuberculosis still remains a major cause of morbidity and mortality worldwide [3].

In 2013, there were an estimated 9.0 million incident cases of Tuberculosis and 1.5 million people died from the disease (1.1 million deaths among people who were HIV-negative and 360000 among people who were HIV-positive). Among these deaths, there were an estimated 210000 from multi drug resistant tuberculosis (MDR- TB), a relatively high total compared with 480000 incident cases of MDR-TB. The South-East Asia and Western Pacific Regions collectively accounted for $56 \%$ of the world's tuberculosis cases in 2013 [2]. The African Region had approximately one quarter of the world's cases, and the highest rates of cases and deaths relative to population, while India and China had the largest number of cases, $24 \%$ and $11 \%$ of the global total respectively [2].

Although the 2015 millennium development goal of 
halting and reversing the TB incidence has been achieved globally, in all six WHO regions and in most of the 22 high TB burden countries, and TB incidence fell at an average rate of about $1.5 \%$ per year between 2000 and 2013 [2], the burden of TB in Nigeria remains high [4]. Also, the burden of both TB and HIV infections in Nasarawa State, Nigeria is also relatively high while TB diagnostics are grossly inadequate [5]. This poses a significant threat to public health and safety in the state, due to population surge and poor environmental and living conditions along with the impact of sexually transmitted diseases. This study therefore seeks to examine the current prevalence rate of tuberculosis and rifampicin resistance amongst suspected patients seeking health care in selected hospitals in Nasarawa state, Nigeria, using microscopy and WHO approved GeneXpert probing technique.

\section{Materials and Methods}

\subsection{Study Area and Population}

The study was conducted in five selected hospitals in Nasarawa State, North central Nigeria. A total of 346 patients seeking medical care for respiratory related disease were included in the study. The patients were drawn from five hospitals as follows: 170 patients from Dalhatu Araf Specialist Hospital (Lafia), 69 patients from Evangelical Reformed Church of Christ (ERCC)-Medical Centre (Alushi), 57 patients from General Hospital (Obi), 44 patients from General Hospital (Nasarawa Eggon) and 6 patients from Federal Medical Centre (Keffi). Management and Ethical approval was obtained from Nasarawa State Ministry of Health (SMOH) Lafia, and the Ethics and Research Committees of the Dalhatu Araf Specialist Hospital, Lafia , Federal Medical Centre Keffi (FMC-Keffi) and ERCC Hospital, before commencement. Also patients consent was obtained before sample collection.

\subsection{Sample Collection and Tuberculosis Detection}

Early morning and on the spot sputum samples were collected in wide mouth capped containers from patients who have not received tuberculosis treatment before or have not received treatment in the past one month. Patients were advised to rinse their mouth first with water to remove particles in the mouth and cautioned not to add saliva to the sputum samples. The samples were properly labeled and transported safely for analysis. Also, relevant sociodemographic data was collected from patients using a structured standard questionnaire.

Sputum Samples were stained directly using Ziehl Neelsen method and slides examined under the microscope using 40x objective to view the distribution of the material and then systematically with the oil immersion (100x) objective to detect Acid fast bacilli (AFB).

\subsection{Rifampicin Resistance Testing}

To confirm the presence of M. tuberculosis and check for resistance to rifampicin, a portion of each positive sputum Sample was subjected to GeneXpert MTB/RIF testing. The GeneXpert MTB/RIF detects DNA sequences specific for $M$. tuberculosis and rifampicin resistance using nucleic acid amplification tests (NAAT). Tests were run following manufacturer's instructions.

\subsection{HIV Status Determination}

Two milliliters $(2 \mathrm{ml})$ of blood samples was collected from all participants using a single sterile syringe and needle for each patient by venipuncture and inserted immediately into an EDTA sample bottle. The Abbot determine HIV $-1 / 2$ test kits (Inverness Medical, Japan) were used for the HIV detection. Positive samples were further confirmed using HIV 1/2 Stat pack (Combio Diagnostic system, USA) as recommended for HIV confirmation.

\subsection{CD4 Counting and Procedure}

CD4 count was determined for HIV positive blood samples using IVD Flow Cytometry (Partec, Germany). Twenty microliter $(20 \mu \mathrm{l})$ of whole blood was added to Partec test tube, $20 \mu 1$ of CD4 mAb PE was added and mixed gently and incubated for 15 mins at room temperature in the dark. Eight hundred microliter $(800 \mu \mathrm{l})$ of lyse buffer was added and shaken or vortexed gently and analyzed using Flow Cytometry (Partec, Germany).

\subsection{Data Collation and Analysis}

Data generated were collated, entered into SPSS version 16 and evaluated using the chi square method at $95 \%$ Confidence limit. The results of the analysis are presented in simple percentages, tables and graph for easy comprehension.

\section{Results}

A total of 346 samples composed of 171 (49.4\%) males and $175(50.6 \%)$ females, were obtained from patients seeking care for respiratory related diseases in Nasarawa State, Nigeria and sampled between February-October, 2015. The overall prevalence of tuberculosis in the study area was $26.3 \%$ with 91 of the 346 samples testing positive for $M$. tuberculosis as shown in table 1 . Out of the 91 positive samples that tested positive for tuberculosis, 52 (57.1\%) were males while $39(42.9 \%)$ were females. There was no statistical relationship between tuberculosis and gender $(\mathrm{P}>$ 0.05 ) (Table 1).

Out of the 91 positive samples, 17 samples were from individuals with no formal education, 27 from individuals whose highest level of education is primary, 30 from individuals with secondary education, 17 with tertiary level of education and 7 with post graduate level of education. Statistically, there was a significant relationship between tuberculosis and level of education $(\mathrm{P}<0.05)$. Also as shown in table 1 , the highest rate of tuberculosis was found within the age range of 25-44 years. Statistically there was no 
significant relationship between tuberculosis and age $(\mathrm{P}>$ $0.05)$. The highest number of the tuberculosis cases was found among farmers, where $34.1 \%$ of those who considered farming as their primary source of income tested positive to TB. This was followed by the civil and public servant, where $33.7 \%$ of those tested were positive for TB. Thirty percent $(30.0 \%)$ of the artisans tested positive, while $22.6 \%$ of the traders were positive (Table 1). These occupations collectively accounted for about $73.6 \%$ of the tuberculosis cases in this study. Statistically there was no significant relationship between tuberculosis and occupation $(\mathrm{P}>0.05)$.

Table 1. Tuberculosis Prevalence by Socio-Demograpiv details.

\begin{tabular}{lll}
\hline Variables & Frequency & Number Positive (\%) \\
\hline Gender & 171 & $52(57.1)$ \\
Male & 175 & $39(42.9)$ \\
Female & & \\
Age & 3 & $0(0.0)$ \\
$>15$ & 72 & $20(27.8)$ \\
$15-24$ & 118 & $29(24.6)$ \\
$25-34$ & 84 & $27(32.1)$ \\
$35-44$ & 48 & $13(27.1)$ \\
$45-54$ & 6 & $1(20.0)$ \\
$55-64$ & 11 & $0(0.0)$ \\
$65-74$ & 4 & $1(25.0)$ \\
$75+$ & & \\
Educational Level & 55 & $17(30.9)$ \\
None formal & 82 & $27(32.9)$ \\
Primary & 103 & $30(29.1)$ \\
Secondary & 99 & $17(17.2)$ \\
Tertiary & 7 & $0(0.0)$ \\
Post Graduate & & \\
Occupation & 51 & $11(21.6)$ \\
student & 104 & $35(33.7)$ \\
Civil \& Public Servant & 62 & $14(22.6)$ \\
Business/Trading & 10 & $3(30.0)$ \\
Artisan & 44 & $15(34.1)$ \\
Farming & 4 & $0(0.0)$ \\
Retired & 70 & $13(18.3)$ \\
None/Unemployed & & \\
\hline
\end{tabular}

The overall prevalence of Rifampicin resistance was $6.6 \%$, with 6 out of the 91 tuberculosis positive samples showing resistant to rifampicin as shown in table 2. The prevalence rate of HIV in Nasarawa state in this study was $46.8 \%, 162$ of the 346 patients tested positive to HIV as shown in table 3 . Out of the 91 samples that tested positive for tuberculosis, 33 $(36.3 \%)$ were HIV positive. Also, $5(83.3 \%)$ out of the 6 samples that showed resistant to rifampicin were HIV positive (Table 4).

Table 2. Prevalence of Tuberculosis and Rifampicin Resistance.

\begin{tabular}{lll}
\hline & Tuberculosis (\%) & Rifampicin resistance (\%) \\
\hline Positive & $91(26.3)$ & $6(6.6)$ \\
Negative & $255(73.7)$ & $85(93.4)$ \\
Total & $346(100)$ & $91(100)$ \\
\hline
\end{tabular}

Table 3. HIV Prevalence.

\begin{tabular}{lll}
\hline HIV Status & Number sampled & Percentage \\
\hline Positive & 162 & 46.8 \\
Negative & 184 & 53.2 \\
Total & 346 & 100.0 \\
\hline
\end{tabular}

Table 4. Prevalence of Tuberculosis and Rifampicin Resistance among HIV Positive patients.

\begin{tabular}{llll}
\hline HIV Status & $\begin{array}{l}\text { Tuberculosis } \\
\text { Positive (\%) } \\
\text { N=91 }\end{array}$ & $\begin{array}{l}\text { Rifampicin } \\
\text { Resistance (\%) } \\
\mathbf{N = 1 6}\end{array}$ & \multirow{2}{*}{$\chi^{2}$-value } \\
\hline HIV Positive & $33(36.3 \%)$ & $5(83.3)$ & \\
HIV Negative & $58(63.7 \%)$ & $1(16.7)$ & \multirow{2}{*}{$\chi^{2}=0.019$} \\
Total & $91(100)$ & $6(100)$ & \\
\hline
\end{tabular}

$\chi^{2}(1, \mathrm{~N}=346)=5.527, \chi^{2}<.05$. Hence statistically, there's a significant relationship between Tuberculosis occurrence and HIV.

From our findings, $28(84.8 \%)$ of the 33 TB-HIV positive patients had CD4 count less than or equal to $300 \mu$ l (Figure 1). After an initial increase from CD4 count range of 1-100 to 101-200, TB occurrence was found to be decreasing with increase in CD4 count. There was no patient with TB-HIV co-infection that had a CD4 count above 500 (Figure 1). Statistically, there was a significant difference between CD4 count and Tuberculosis occurrence $(\mathrm{P}<0.05)$.

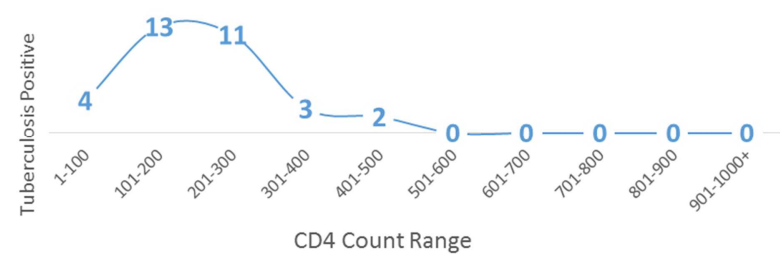

Figure 1. Prevalence of Tuberculosis by CD4 Count Range.

\section{Discussion}

Tuberculosis continues to occupy a prominent place among the major infectious diseases in underdeveloped and developing countries. The increase in tuberculosis cases has mostly been due to the emergence of AIDS in the 1980s, together with factors of impoverishment, social disorder, and lack of investment in effective disease control programs [6].

This study was design to determine the prevalence of $M$. tuberculosis, rifampicin resistance and HIV, in patients seeking medical care services in five selected health facilities in Nasarawa State, North central geopolitical zone of Nigeria. Our findings show that $26.3 \%$ of the patients tested positive to TB. A study carried out earlier in Niger state, which is in the same North central geopolitical zone, reported a higher prevalence of TB [7]. The finding that $36.3 \%$ of HIV positive patients had TB is very close to the $34.5 \%$ reported by Gyar et al. [8] among HIV positive individuals in Lafia, Nasarawa state but less than $52.7 \%$ reported in Niger state [7].

The findings of this research that men are more prone to TB than women agree with WHO report of higher TB rate among men than women [2]. Also Nyamogoba et al. [9], reported a higher tuberculosis rate in males in Kenya. However, the findings in this study of a high rate of tuberculosis among women (47.3\%), greater than the expected 3:1 ratio, only seem to highlight WHO concern of increasing TB burden in women. There is lack of information explaining the role of gender in M. tuberculosis transmission 
and why TB seems to occur more in men than women. The findings of this research that the highest number of Tuberculosis cases occurred among farmers $34.1 \%$ and civil/public servants $33.7 \%$ negates the finding of Gyar et al. [8], who had the least number of positive cases $21.7 \%$ amongst civil servants but agrees with their findings of a high tuberculosis prevalence among those involve in business/trading and the unemployed including house wives. When considered from the level of education, there was not much difference in the prevalence of tuberculosis among those with no formal education up to those with secondary education, but the percentage significantly reduced for those with tertiary level of education. None of the 7 people with post graduate level of education tested positive for TB.

Accurate, rapid detection of M. tuberculosis and TB drug resistance is critical for improving patient care and decreasing TB transmission. Xpert ${ }^{\circledR}$ MTB/RIF assay is an automated test that can detect both $\mathrm{TB}$ and rifampicin resistance, generally within two hours after starting the test [10]. Rifampicin is an important drug for treating people with TB [10]. Therefore Xpert ${ }^{\circledR}$ MTB/RIF assay was used in detecting Rifampicin resistance. Rifampicin resistance of $6.6 \%$ found in this study is very close to the $7.0 \%$ reported in samples collected from Jos and Lagos, Nigeria [11]. Another study in Calabar Nigeria reported a rifampicin resistance of $6 \%$, although they did not find any patient with resistance to only rifampicin [12]. Using BACTEC 960 system for drug susceptibility testing in Abuja, Lawson et al. [13] reported that $10(31 \%)$ confirmed tuberculosis samples were resistant to at least one of the four drugs tested including rifampicin, while $4(13 \%)$ were resistant to the four drugs (isoniazid, ethambutol, streptomycin and rifampicin) tested and were classified as multidrug resistant-TB (MDR-TB). Globally, $3.5 \%$ of new TB cases and $20.5 \%$ of previously treated cases are estimated to have MDR-TB, while the estimated percentage of new Tuberculosis with MDR-TB in Nigeria is 2.9 [2].

Tuberculosis and HIV co-infection rate was found to be $36.3 \%$ as presented in table 4 . Reports of similar studies in Nigeria found $12.0 \%$ in Ile-Ife [14], $10.0 \%$ in Kano [15], $10.5 \%$ and $14.9 \%$ among children and adults respectively in Sagamu [16, 17], and $41.2 \%$ in Alushi [18]. Elsewhere, Akpaka et al. [19] reported 11.6\% TB and HIV co-infection in Jamaica. Also, 11.4\% was found in Northwest Ethiopia [20], 55.5\% in Western Kenya [9], 39.0\% in Brazil [21] and $8.6 \%$ in India [22]. The fairly high prevalence of tuberculosis disease among HIV/AIDS patients seeking care and treatment in the selected health facilities in this research suggests a strong association between TB and HIV infection as posited by Tadesse and Tadesse [20]. This study did not consider whether or not the HIV patients were already on Anti-Retroviral therapy, which may explain why the percentage of TB and HIV co-infection is higher than most of the other figures within Nigeria. Infection with human immunodeficiency virus (HIV) is found to increase the occurrence of drug resistant tuberculosis [23]. This is in agreement with our finding that $5(83.3 \%)$ of the 6 samples that showed rifampicin resistance were from HIV positive patients.

The total number of circulating CD4 cells in HIV infected patients have largely been acknowledged as the strongest, single predictive factor of clinical deterioration [24, 25]. In individuals with latent Mycobacterium tuberculosis infection, CD4 depletion accelerates the progression from latent infection to active tuberculosis (TB), which, in turn, is believed to further fuel HIV replication rates due to elevated levels of pro-inflammatory cytokines [26]. This is reflected in our findings which showed an inverse relationship between the CD4 count and presence of M. tuberculosis in HIV positive patients. As the number of CD4 count was increasing in this group of patients, the number positive for TB was reducing. TB was not detected in patients with CD4 count of 500 and above.

\section{Conclusion}

Our findings have shown that tuberculosis still remain endemic in Nasarawa state with a high prevalence of HIV. The confluence of HIV and tuberculosis increased the rate of rifampicin resistance. Further studies that will include more antibiotics are highly recommended.

Limitation: This study did not include resistance to isoniazid because the Xpert cartridge for it was not available.

\section{References}

[1] World Health Organization. "Global tuberculosis control". Surveillance, planning, financing Rep WHO/HTM/TB/2008393geneva: World Health Organization Report, 2013.

[2] World Health Organization (2014). "Global Tuberculosis Report," 2014. Retrieved from: www.who.org

[3] E. L. Corbett, C. J. Watt, N. Walker, D. Maher, and B. G. Williams, "The growing burden of tuberculosis: global trends and interactions with the HIV epidemic," Arch. Internal Med, vol. 163, pp. 1009-1021, 2003.

[4] C. C. Dim, and N. R. Dim, "Trends of tuberculosis prevalence and treatment outcome in an under-resourced setting: The case of Enugu state, South East Nigeria," Nig Med J, vol. 54, pp. 392-397, 2013.

[5] B. N. Joseph, and E. S. Fanisi, "Assessing Treatment outcomes in previously treated Tuberculosis Patient's in Nasarawa State, Nigeria: Implication for Resistance," Merit Res J Med Medical Sc, vol. 3, pp. 130-134, 2015.

[6] M. V. Souza, "Tuberculose empacientes HIV-positivos, um grave problema de saúdemundial," Rev Bras Farm, vol. 87, pp. 42-44, 2006.

[7] K. Ibrahim, O. O. Akanni, and U. J. Ijah, "The prevalence of tuberculosis in HIV patients in Minna metropolis," Nig J Microbiol, vol. 18, pp. 212-216, 2004.

[8] S. D. Gyar, E. Dauda, and C. R. Reuben, Prevalence of tuberculosis in HIV/AIDS patients in Lafia, central Nigeria. Int J Curr Microbiol Appl Sc, vol. 3, pp. 831-838, 2014. 
[9] H. D. N. Nyamogoba, G. Mbuthia, G. Kikuvi, S. Mpoke, and P. G. Waiyaki, "A high tuberculosis and human immunodeficiency virus co-infection rate and clinical significance of non-tuberculous mycobacteria in Western Kenya,” Afr J Health Sc, Vol. 21, pp. 147-154, 2012.

[10] K. R. Steingart, I. Schiller, D. J. Horne, M. Pai, C. C. Boehme, and N. Dendukuri, "Xpert® MTB/RIF assay for pulmonary tuberculosis and rifampicin resistance in adults," Cochrane Database of Systematic Reviews, Issue 1, 2014 Art. No.: CD009593. DOI: 10.1002/14651858.CD009593.pub3.

[11] L. Dinic, P. Akande, E. O. Idigbe, A. Ani, D. Onwujekwu, O. Agbaji, M. Akanbi, R. Nwosu, B. Adeniyi, M. Wahab, C. Lekuk, C. Kunle-Ope, N. Nwokoye, and P. Kanki, "Genetic determinants of drug-resistant tuberculosis among HIVinfected patients in Nigeria," J Clin Microbiol, Vol. 50, pp. 2905-2909, 2012.

[12] A. Otu, V. Umoh, A. Habib, S. Ameh, L. Lawson, and V. Ansa, "Drug Resistance among Pulmonary Tuberculosis Patients in Calabar, Nigeria," Pulmonary Med 2013, E1-7, 2013.

[13] L. Lawson, A. G. Habib, M. I. Okobi, D. Idiong, I. Olajide, N. Emenyonu, N. Onuoha, L.E. Cuevas, and S.O. Ogiri, "Pilot study on multidrug resistant tuberculosis in Nigeria," Ann Afr Med, vol. 9, pp. 184-187, 2010.

[14] A. O. Onipede, O. Idigbe, A. K. Ako-Nai, O. Omojola, A. O. Oyelese, A. O. Aboderin, A. O. Komolafe, and S. N. C. Wemambu, "Seroprevalence of HIV antibodies in TB patients in Ile-Ife," Afr Med J, vol. 76 pp. 127-132, 1999.

[15] Z. Iliyasu, and M. Babashani, "Prevalence and Predictors of TB coinfection Among HIV seropositive patients attending Aminu Kano Teaching Hospital, Northern Nigeria," J. Epid, vol. 2 pp. 81-87, 2009.

[16] O. J. Daniel, A. A. Salako, F. A. Oluwole, O. K. Alausa, and O. T. Oladepo, "HIV seroprevalence among newly diagnosed adult PTB patients in Sagamu" Nig J Med, vol. 13, pp. 393$397,2004$.

[17] O. J. Daniel, O. B. Ogunfowora, and O. T. Oladapo, "HIV seroprevalence among Children diagnosed with $\mathrm{TB}$ in Nigeria," Trop Doctor, vol. 37, pp. 268-269, 2007.

[18] G. Pennap, S. Makpa, and S. Ogbu, "The Prevalence of HIV/AIDS among Tuberculosis Patients in a Tuberculosis/Leprosy Referral Center in Alushi, Nasarawa State, Nigeria" The Internet J Epid, vol. 8 no.1, 2009.

[19] P. E. Akpaka, M. Tulloch-Reid, A. Justiz-Vaillant, and M. F. Smikle, "Prevalence of human immunodeficiency virus infection in patients with pulmonary tuberculosis at the National Chest Hospital in Jamaica," Rev Panam Salud Publ, vol. 19, pp. 38-43, 2006.

[20] S. Tadesse, and T. Tadesse, "HIV co-infection among tuberculosis patients in Dabat, northwest Ethiopia," J Infect Dis Immun, vol. 5, pp. 29-32, 2013.

[21] M. Santos Neto, F. L. Silva, K. R. Sousa, M. Yamamura, M. P. Popolin, and R. Arcêncio, "Clinical and epidemiological profile and prevalence of tuberculosis/HIV co-infection in a regional health district in the state of Maranhão, Brazil," J. Bras. Pneumol, vol. 38, pp. 724-732, 2012.

[22] R. C. Rajam Mano and G. M. Muhammad, "A Study on HIV co-infection among pulmonary tuberculosis at a private medical college hospital," Asian Student Med J, vol. 13, pp. 111, 2013.

[23] S. Rajasekaran, C. Chandrasekar, A. Mahilmaran, K. Kanakaraj, D. S. Karthikeyan, and J. Suriakumar, "HIV coinfection among multidrug resistant and extensively drug resistant tuberculosis patients-a trend," J Indian Med Assoc, vol. 107, pp. 281-286, 2009.

[24] K. Begtrup, M. Melbye, R. J. Biggar, J. J. Goedert, K. Knudsen, and P. K. Andersen, "Progression to acquired immunodeficiency syndrome is influenced by CD4 Tlymphocyte count and time since seroconversion," Am J Epid, vol. 145 , pp. 629-635, 1997.

[25] World Health Organization, "Global tuberculosis control" WHO report, 2010.

[26] J. J. Ellner, "Immunoregulation in TB: observation and implications," Clin Trans Sc, vol. 3, pp. 23-28, 2010. 